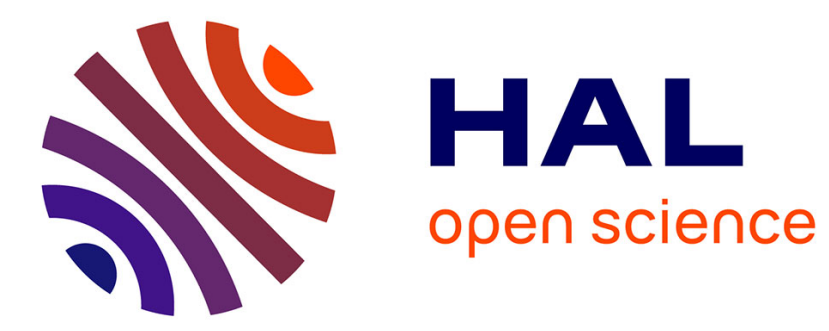

\title{
Modeling force transmission in granular materials Farhang Radjai
}

\section{To cite this version:}

Farhang Radjai. Modeling force transmission in granular materials. Comptes Rendus. Physique, 2015, 16 (1), pp.3-9. 10.1016/j.crhy.2015.01.003 . hal-02094888

\section{HAL Id: hal-02094888 \\ https://hal.science/hal-02094888}

Submitted on 10 Apr 2019

HAL is a multi-disciplinary open access archive for the deposit and dissemination of scientific research documents, whether they are published or not. The documents may come from teaching and research institutions in France or abroad, or from public or private research centers.
L'archive ouverte pluridisciplinaire HAL, est destinée au dépôt et à la diffusion de documents scientifiques de niveau recherche, publiés ou non, émanant des établissements d'enseignement et de recherche français ou étrangers, des laboratoires publics ou privés. 


\title{
Modeling force transmission in granular materials
}

\section{Modélisation de la transmission des forces dans les matériaux granulaires}

\author{
Farhang Radjai a,b,* \\ a LMGC, UMR 5509 CNRS - University of Montpellier, place Eugène-Bataillon, 34095 Montpellier, France \\ ${ }^{\mathrm{b}}$ MultiScale Material Science for Energy and Environment, UMI 3466 CNRS-MIT, CEE, Massachusetts Institute of Technology, 77 \\ Massachusetts Avenue, Cambridge 02139, USA
}

Keywords:

Granular matter

Force chains

Arching

Contact dynamics

Molecular dynamics

Mots-clés:

Matière granulaire

Chaînes de force

Effet de voûte

Exclusions stériques

Dynamique des contacts

Dynamique moléculaire

\section{A B S T R A C T}

The probability density function of contact forces in granular materials has been extensively studied and modeled as an outstanding signature of granular microstructure. Arguing that particle environments play a fundamental role in force transmission, we analyze the effects of steric constraints with respect to force balance condition and show that each force may be considered as resulting from a balance between lower and larger forces in proportions that mainly depend on steric effects. This idea leads to a general model that predicts an analytical expression of force density with a single free parameter. This expression fits well our simulation data and generically predicts the exponential fall-off of strong forces, a small peak below the mean force and the non-zero probability of vanishingly small forces.

\section{R É S U M É}

La densité de probabilité des forces de contact dans les matériaux granulaires représente une signature remarquable de la microstructure granulaire et, à ce titre, elle a fait l'objet de nombreuses études et d'efforts de modélisation. Nous allons analyser le rôle fondamental des environnementaux locaux des particules pour la transmission des forces et les effets des contraintes stériques par rapport à l'équilibre des forces. Cette analyse permet de montrer qu'une force de contact met en jeu des forces supérieures et inférieures à cette force dans des proportions qui sont contrôlées par les effets stériques. Cette idée simple conduit à un modèle général qui prédit une expression analytique de la densité des forces avec un seul paramètre libre. Ce paramètre coïncide avec le degré d'homogénéité des forces et peut dépendre de l'anisotropie du réseau des contacts ou des formes et distributions des tailles des particules. Cette expression ajuste bien les données numériques et prédit d'une manière générique la décroissance exponentielle des forces fortes, un petit pic en dessous de la force moyenne et une densité de probabilité non nulle pour les forces très petites.

\footnotetext{
* Correspondence to: LMGC, UMR 5509 CNRS - University of Montpellier, place Eugène-Bataillon, 34095 Montpellier, France. E-mail address: franck.radjai@univ-montp2.fr.
} 

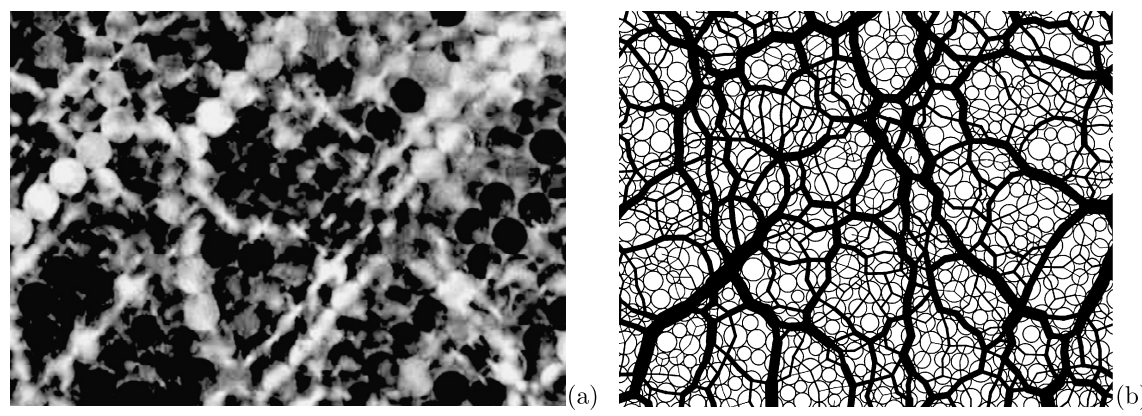

Fig. 1. (a) Photoelastic image of an assembly of birefringent spheres [18]. (b) Map of normal forces in a simulated packing of disks. Line thickness is proportional to normal force.

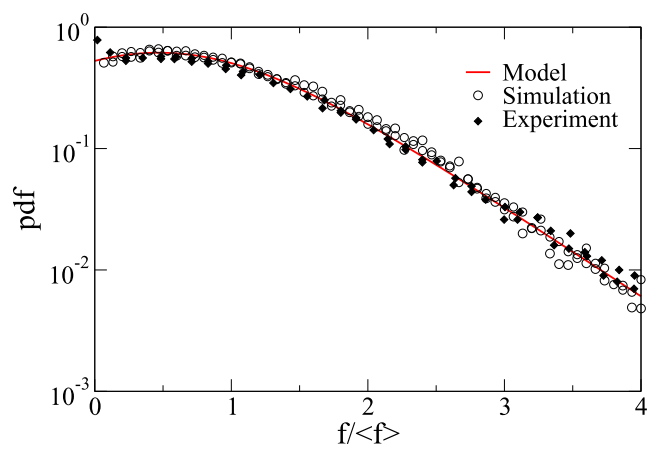

Fig. 2. (Color online.) Probability density function of normal contact forces normalized by the mean force in static granular materials from simulations and experimental data of Mueth et al. [8]. The solid line is the prediction of the model introduced in this paper yielding expression (10).

\section{Introduction}

Geometrical disorder and steric exclusions in granular materials lead to an unexpectedly inhomogeneous distribution of contact forces [1-13]. The inhomogeneous transmission of stresses was initially observed by means of photoelastic experiments with particles made of a birefringent material [1,2]. A photoelastic image, such as that displayed in Fig. 1(a), readily reveals filaments of bright grains subjected to high loads against a background of dark zones composed of much less loaded grains. Such filamentary patterns of stresses inside grains are induced by strong contact forces as those shown in Fig. 1(b) from numerical simulations, and are known as force chains. The linear aspect of chains observed in most reported experiments reflects long-range ordering of grains of nearly the same size in a 2D geometry. But this linear aspect of strong force chains is more generally observed in simulations of arbitrary grain shapes and size distributions in 2D and 3D, in which a variety of other patterns such as arch-type chains are quite common [10,14-17].

The forces were measured for the first time by using carbon paper to record normal force prints at the boundaries of a bead packing $[4,8]$. They were found to have a nearly uniform probability density function (pdf) in the range of weak forces followed by an exponential falloff of strong forces. Similar force distributions were found by later experiments $[7,19]$ using contact area trace and by means of numerical simulations [5,6,20,10,11,21]. Fig. 2 shows a typical force density obtained by two different numerical methods for the same packing built by isotropic compaction as well as the data obtained by the experiments of Mueth et al. by means of carbon paper trace [8]. The data from our simulations and experiments coincide every where within the available precision in exception to the range of vanishingly small forces, where the details of sample preparation matter. The solid line is the plot of expression (10) predicted by the model that will be presented in this paper.

Detailed analysis of sheared granular materials provided further evidence for the bimodal organization of the force network in well-defined weak and strong networks with the strong network contributing almost exclusively to the shear strength and weak forces acting mainly to prop strong force chains [22]. All further investigations of force distributions have shown that the exponential distribution of strong forces is a robust feature of force distribution in granular media both in two and three dimensions. In contrast, the weak forces appear to be sensitive to the packing state resulting from the deformation history $[21,23,24]$. In an isotropic packing state, the distribution shows a relatively small peak below the mean force as seen in Fig. 2, and the probability density of small forces does not fall to zero [25,26]. The peak disappears in a sheared packing and the distribution of weak forces turns to a nearly decreasing power law $[10,21,27]$. This is also what generally is observed in frictional packings composed of aspherical grains or broad size distributions $[28,15,17,29-31]$.

The sensitivity of force probability density to packing states and grain characteristics indicates that it can be used as structural descriptor of granular materials, and it might even be more relevant to rheology of granular materials than purely geometrical descriptors such as radial pair distributions [32]. In this sense, a successful modeling approach may reveal 


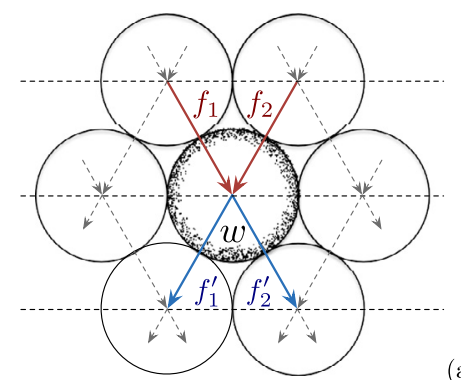

(a)

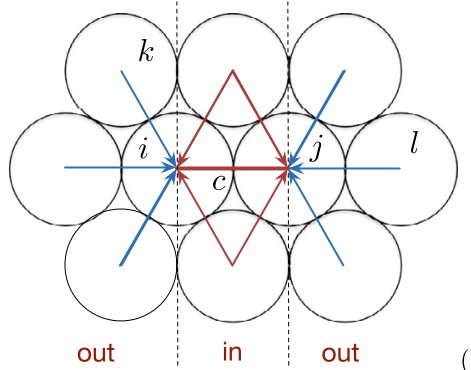

(b)

Fig. 3. (Color online.) (a) "Propagation" of forces in the $q$-model. (b) Local environment of a contact $c$ in an ordered packing.

the respective roles of disorder, steric exclusions (with the effect of constraining grain environments) and force balance in the rheological behavior of granular materials. The $q$-model was the first attempt in this direction [4,33]. The forces are assumed to be scalar quantities that "propagate" from site to site (particles) along the links (contacts) of a regular network, as illustrated in Fig. 3(a). The total incoming force $w=f_{1}+f_{2}$ from the particles of a layer to each site is redistributed to the particles of the next layer according to a random process: $f_{1}^{\prime}=q w$ and $f_{2}^{\prime}=(1-q) w$ where $q$ is a random variable $\in[0,1]$. It is further assumed that the incoming forces are not correlated so that $P\left(f_{1}, f_{2}\right)=P\left(f_{1}\right) P\left(f_{2}\right)$ in analogy with 'molecular chaos' assumption in kinetic theory of gases.

The $q$-model predicts that the force pdf converges to a purely exponential function $P(f)=\beta \mathrm{e}^{-\beta f}$ with the exponent $\beta$ depending on the number of supporting contacts per grain. A typical value $\simeq 1.5$ is obtained by fitting the range of strong forces in Fig. 2. This value is, however, almost independent of space dimension, which controls the number of contacts per grain, thus contradicting the $q$-model. The pdf of $w$ is of the form $P(w)=\beta^{2} w \mathrm{e}^{-\beta w}$ (being often confused with $P(f)$ ). A common belief is that this model may apply to the components $f_{x}$ of the forces along a direction $x$ in a truly disordered system. But unfortunately, the angular hindrances between the particles lead to a strong correlation of force components and thus the molecular chaos assumption does not hold. Later efforts to generalize this model to vectorial force balance have not led to analytical forms. But a statistical approach developed by Metzger et al. provides a correct estimate of the force pdf similar to Fig. 2 by analyzing the local constraints and accounting for the density of states in a first-shell approximation (one grain with its contact neighbors) [26].

It is worth mentioning here the developments referring to the entropy of contact forces in a 'virtual' thermodynamics to rationalize the exponential distribution of strong forces as a consequence of the density of force states imposed by boundary stresses [34]. Such an appealing analogy with the Maxwell-Boltzmann distribution of velocities does not recover the nonzero probability of vanishing forces but it suggests that the exponential distribution of strong forces is not a decisive test for force models as it arises from a general statistical argument. Other routes have successfully been explored to obtain semi-analytical fits to the force pdf in isotropic dense packings. For example, a mathematical analysis was developed on the relationship between the force pdf's and their directional components [35]. This analysis indicates that, if the forces in $q$-model are the components of contact forces along the propagation direction, the resulting distribution for the normal forces is a Bessel function passing through the origin, and, in this respect, it does not correctly model the range of weak forces. But a linear combination of several functions provides a convenient fit to the data points.

In this paper, we introduce a new model that leads to an analytical expression of force probability density. We first discuss the effects of steric exclusions on force balance conditions in a contact environment. We argue that a force may occur at a given contact depending on the availability of forces above that force in the neighboring contacts as well as smaller forces allowing for static equilibrium. This idea can be cast into an integral-differential equation, which has a simple analytical solution predicting an exponentially decreasing tail, a small peak the mean force and a nonzero force probability at zero force. This expression fits numerical and experimental data by adjusting a single free parameter of the model.

\section{Force balance and steric constraints}

The pdf of contact force magnitudes (normal or tangential) are 'atypical' in the sense that they differ from what may be expected from intuition. Physically, the forces are repulsive reactions between particles as a result of elastic deflections at their contact points. But in the absence of a characteristic internal force (assuming no cohesive force), the contact forces should scale with boundary stresses. The simplest configuration is a granular packing in static equilibrium under the action of an isotropic pressure $p$. It can be shown that, independently from grain stiffness, the mean force $\langle f\rangle$ is given by

$$
\langle f\rangle=\frac{D p}{n_{\mathrm{c}} \ell}
$$

where $D$ is space dimension, $n_{\mathrm{c}}$ is the number density of contacts and $\ell$ is the mean branch vector length (vector joining grain centers). The mean contact deflection can be obtained from this expression and a pressure dependent effective elastic modulus $E^{*}(p)$. Hence, it is expected that all contact forces scale with the mean $\langle f\rangle$ and their pdf is also centered on the mean. However, while the forces do scale with the mean force, the force pdf's lack central tendency or show only a small 
mode below the mean. Moreover, the forces below the mean down to vanishingly small forces have larger density than the mean force, as observed in Fig. 2. Simulations indicate that more than $60 \%$ of contacts are in this range.

Qualitatively, the so-called arching effect is relevant to this behavior. Not only a finite proportion of particles ( $\simeq 5 \%$ in the isostatic state of a weakly polydisperse system [36]) is always excluded from the force-bearing contact network, but also many local configurations of the grains can be seen as micro-arches that screen many much less loaded grains. This is a vectorial effect and cannot be captured by a scalar model of force transmission. In fact, a micro-arch occurs since, due to high packing fraction, an arch-like structure can be equilibrated by forces acting along different contact directions much as in an architectural arch. To build upon this picture, we need to focus on the contacts rather than particles.

Let us consider two particles $i$ and $j$ inside a packing touching at a contact $c$ as illustrated in Fig. 3(b). For clarity, we consider a regular packing with zero friction coefficient. The static equilibrium of the two particles is governed by the following equations:

$$
\sum_{k \in i} f_{i k} \vec{n}_{i k}=0 \text { and } \sum_{l \in j} f_{i l} \vec{n}_{i l}=0
$$

where $k$ and $l$ denote the contacts belonging to $i$ and $j$, respectively. The force $f_{i k}$ (resp. $f_{j l}$ ) is exerted by contact neighbors of particle $i$ (resp. $j$ ) along the contact direction $n_{i k}$ (resp. $n_{j l}$ ) pointing inward. With these sign conventions, all forces $f_{i k}$ and $f_{j l}$ are positive. Multiplying both equations by $\vec{n}_{j i}$ and subtracting the first equation from the second one, we get

$$
f_{\mathrm{c}} \equiv f_{i j}=f_{\mathrm{c}}^{\text {out }}-f_{\mathrm{c}}^{\text {in }}
$$

with

$$
\begin{aligned}
& f_{\mathrm{c}}^{\text {out }}=\frac{1}{2} \sum_{k \neq j}\left(-f_{i k} \vec{n}_{i k} \cdot \vec{n}_{i j}\right)^{+}+\frac{1}{2} \sum_{l \neq i}\left(-f_{j l} \vec{n}_{j l} \cdot \vec{n}_{j i}\right)^{+} \\
& f_{\mathrm{c}}^{\text {in }}=\frac{1}{2} \sum_{k \neq j}\left(f_{i k} \vec{n}_{i k} \cdot \vec{n}_{i j}\right)^{+}+\frac{1}{2} \sum_{l \neq i}\left(f_{j l} \vec{n}_{j l} \cdot \vec{n}_{j i}\right)^{+}
\end{aligned}
$$

where $(\ldots)^{+}$refers to the positive part of $(\ldots)$. The force $f^{\text {out }}$ is the sum of all forces acting by their components along $n_{i j}$ to push one particle against the other whereas $f^{\text {in }}$ is the sum of force components tending to move apart the two particles. We refer to these forces as 'in' and 'out' forces, respectively, as they are exerted by the neighboring particles lying in the angular space between the two particles in the case of 'in' forces and by the neighboring particles lying in the outer parts of the two particles in the case of 'out' forces.

The "force transfer equations" (3) for all contacts $c$ in a packing are equivalent to the force balance equations for the particles. They have the advantage of suggesting a relaxation method for computing the contact forces. Start with a set of arbitrary values $f_{\mathrm{c}}$ of all normal forces. From these values and given boundary forces, compute the values of $f_{\mathrm{c}}^{\text {in }}$ and $f_{\mathrm{c}}^{\text {out }}$ and thus $f_{\mathrm{c}}^{\prime}=f_{\mathrm{c}}^{\text {out }}-f_{\mathrm{c}}^{\text {in }}$ for each contact. The calculated value $f_{\mathrm{c}}^{\prime}$ of the force at contact $c$ replaces $f_{\mathrm{c}}$ only if it is positive. Otherwise, $f_{\mathrm{c}}$ should be set to zero. The same operation can be repeated with the new values of forces. This iteration process between $f_{\mathrm{c}}$ and $\left(f_{\mathrm{c}}^{\text {in }}, f_{\mathrm{c}}^{\text {out }}\right)$ via transfer equations converges to a solution within a given precision level $\left|f_{\mathrm{c}}-f_{\mathrm{c}}^{\prime}\right|<\varepsilon$ if such a solution of static equilibrium exists. A similar transfer equation can also be derived for tangential forces.

The sign condition $f_{\mathrm{c}} \geq 0$ is a major constraint that imposes $f_{\mathrm{c}}^{\text {in }} \leq f_{\mathrm{c}}^{\text {out }}$. An exact compensation between $f_{\mathrm{c}}^{\text {in }}$ and $f_{\mathrm{c}}^{\text {out }}$ implies $f_{\mathrm{c}}=0$. In other words, the contact $c$ transmits no force and thus the particles $i$ and $j$ are connected through other contacts in the neighborhood of contact $c$. If the transfer equations are written from the equations of dynamics for both particles, as in the contact dynamics method [37,38], the relative velocity of the two particles at the contact point can be obtained through a similar relaxation method.

The contact environment shown in Fig. 3(b) together with its transfer equation highlights the role of steric exclusions in force balance. First, the angular hindrance among neighboring particles in the vicinity of contact $c$ imply significant angular ordering even for a polydisperse size distribution. Secondly, as a result of the vectorial nature of static equilibrium and inevitable steric angles, branching is not an efficient mechanism for the splitting of forces into smaller forces. For particles of the same size, force balance can be trivially achieved by simply setting all forces equal to the mean force (equivalently, with $f_{\mathrm{c}}^{\text {in }}=\langle f\rangle$ and $\left.f_{\mathrm{c}}^{\text {out }}=2\langle f\rangle\right)$. From this picture, it is by no means evident where the variability of the random parameter $q$ in $q$-model arises from. The variations of the angular positions due to polydispersity affect only slightly $f_{\mathrm{c}}^{\text {in }}$ and $f_{\mathrm{c}}^{\text {out }}$ due to the projections $\vec{n}_{i k} \cdot \vec{n}_{i j}$ (resp. for particle $j$ ). In contrast, a connectivity disorder due to "missing" contacts has a drastic effect. In this respect, the granular disorder is mainly characterized by the fluctuations of local environments due to connectivity. This image is still partly correct in highly polydisperse granular materials as strong force chains are mostly captured by large particles [28]. Note that we have as many transfer equations as the number of contacts but all equations are not independent. The degree of indeterminacy can be calculated by evaluating the null space of the operator that transforms force balance equations into transfer equations [39]. The set of solutions compatible with the contact network may be modeled as a statistical ensemble with a flat measure [40]. But it is also important to remark that the forces cannot be analyzed independently from their history and part of the memory of a granular material is encoded in contact forces, 

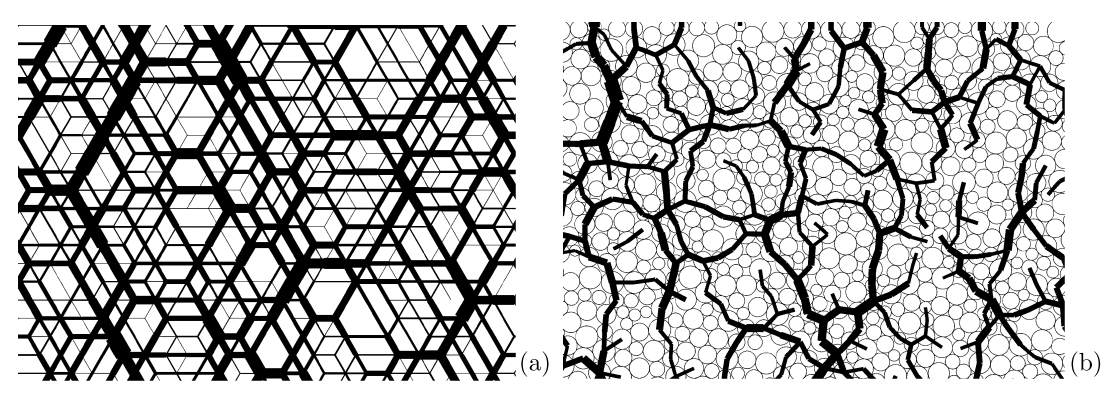

Fig. 4. (a) Snapshot of normal forces computed by an iterative process on a lattice with random connectivity of the sites. (b) Normal forces above the mean force in a simulated packing. Line thickness is proportional to force magnitude.

and more specifically in friction mobilization when the coefficient of friction is nonzero. The force ensemble statistics may thus be considered as a way of determining the forces by removing arbitrary bias in the choice of forces.

Assuming connectivity randomness in particle environments (with the coordination number varying from one particle to another), we naturally arrive at a force line picture: Large forces 'propagate' across particles connecting nearly parallel contacts along a direction until, due to a missing contact in that direction, branching occurs into at least two different directions. This is what we basically observe in Fig. 4(a) where the forces are simulated by means of transfer equations on a triangular lattice from which a fraction of contacts are removed (by imposing a zero force at those contacts). The force pdf in this system is similar to that shown in Fig. 2. Fig. 4(b) displays the forces above $\langle f\rangle$ in a polydisperse sample simulated by the contact dynamics method. The observed force lines indicate that large forces occur mostly in linear patterns that end with branching or by progressive attenuation. Apart from rare exceptions, each force is generated from larger forces. But according to transfer equations, arbitrarily small forces can arise from arbitrarily large forces since the force at a contact is given by a difference between 'out' and 'in' forces. Hence, the weak forces are required to restore static equilibrium in the presence of large forces.

\section{Force cascade model}

The model that we present in this section is inspired by the above qualitative picture based on a cascading process from top (large force) to bottom (small force). A force $f$ can be born from the splitting of an arbitrary force $f^{\prime} \geq f$ in its local environment but the reverse is not true, i.e. $f$ cannot be generated from the combination of arbitrarily small forces. Nevertheless, small forces are required to balance large forces, which, as observed in Fig. 4, are not self-balanced. Hence, we postulate that the probability density $P(f)$ of force $f$ is proportional to the probability $G(f)=\int_{f}^{\infty} P(x) \mathrm{d} x$ of forces above $f$. The fraction $b G(f)$ of forces, where $b$ is the factor of proportionality, represents the force chains from which the force $f$ is born. Any of the remaining forces $(1-b) G(f)$ above $f$ and smaller forces $1-G(f)$ may prop the force $f$ to restore static equilibrium. We assume that the probability density $P(f)$ is thus also proportional to $1-b G(f)$. Since the forces are expected to scale with the mean force $\langle f\rangle$, we also assume that all forces are normalized by $\langle f\rangle$. As a result, the probability density of forces is governed by

$$
P(f)=a b G(f)[1-b G(f)]
$$

with

$$
G(f)=\int_{f}^{\infty} P(x) \mathrm{d} x, \quad \int_{0}^{\infty} P(f) \mathrm{d} f=1 \quad \text { and } \quad \int_{0}^{\infty} f P(f) \mathrm{d} f=1
$$

$a$ being a normalization factor.

The normalization condition implies $G(0)=1$, and from Eq. (7) we have $\mathrm{d} G / \mathrm{d} f=-P(f)$. Inserting the latter in (6), we get the following equation for $G$ :

$$
\frac{\mathrm{d} G}{\mathrm{~d} f}=-a b G(1-b G)
$$

This equation is easily solved to yield the following solution:

$$
G(f)=\frac{1}{b+(1-b) \mathrm{e}^{a b f}}
$$

from which the normalized probability density function of forces is obtained:

$$
P(f)=\beta(1+\gamma) \frac{\gamma \mathrm{e}^{\beta f}}{\left(1+\gamma \mathrm{e}^{\beta f}\right)^{2}}
$$




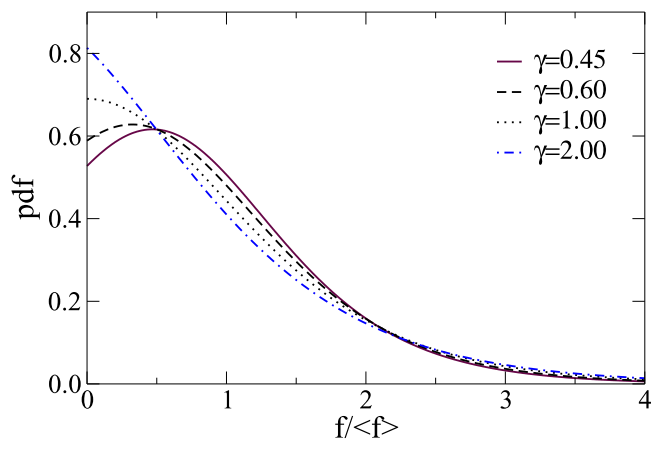

Fig. 5. (Color online.) Plots of the probability function $P(f)$ given by Eq. (10) for different values of the model parameter $\gamma$.

with $\beta=a b$ and $\gamma=1 / b-1$. In this form, $P(f)$ is a normalized function with two parameters. But imposing that the mean force is 1 , we get the following relation between the two parameters:

$$
\beta=(1+\gamma) \ln \left(\frac{1+\gamma}{\gamma}\right.
$$

With this relation, expression (10) represents the pdf of normalized forces with a single free parameter $\gamma$ or $\beta$.

Fig. 5 shows the shape of this function in the range $f \geq 0$ for four values of $\gamma=0.45,06,1$ and 2 , which according to relation (11) correspond to $\beta=1.7,1.57,1.38$ and 1.22 , respectively. The probability density for vanishingly small forces is $P(0)=\gamma \ln [(1+\gamma) / \gamma]$ and it increases with $\gamma$. A peak occurs at $f=-\ln \gamma /\{(1+\gamma) \ln [(1+\gamma) / \gamma]\}$ for $\gamma \leq 1$. It occurs at $f=0$ for $\gamma=1$ and shifts to the right as $\gamma$ decreases. It is also easy to see that $P$ tends to an exponential function $\beta[(1+\gamma) / \gamma] \mathrm{e}^{-\beta f}$ as $f$ increases.

The fitting form displayed in Fig. 2 is the plot of the function (10) with $\beta=1.7$ (i.e. $\gamma=0.45$ ). We see that it is in excellent agreement with numerical and experimental data. Note that a nearly flat density of weak forces, which was observed in the first simulations, corresponds to $\gamma=1$ with a peak at $f=0$ and $\beta=2 \ln 2 \simeq 1.38$. Remarkably, this value coincides with the value $\simeq 1.4$ that was reported for the exponent of the exponential falloff of strong forces when a nearly flat distribution of weak forces was observed [5]. The probability density in this limit takes the simpler form $P(f)=$ $\beta /\left[2 \cosh ^{2}(\beta f)\right]$.

The force cascade model captures quite naturally both the exponential distribution of strong forces and the unmonotonic distribution of weak forces. The predicted family of force functions is defined by a single free parameter $\gamma$. Hence, the effects of various parameters such as friction between particles or force network anisotropy should always express themselves through this same parameter. For example, the friction between particles leads often (depending on the deformation history) to a lower coordination number $Z$ and thus a higher force inhomogeneity. In the framework of the force cascade model, this implies a larger proportion $1-b G(f)$ of smaller forces involved and thus smaller value of $b=1 /(1+\gamma)$ and larger value of $\gamma$. The contact network anisotropy has a similar effect. It often takes larger values for lower values of $Z$ [41], implying a larger value of $\gamma$. Typically, at high enough anisotropy, the force pdf is a decreasing function in the range of weak forces [21]. In Eq. (10), this happens for $\gamma>1$ and the force pdf tends to a purely exponential function as $\gamma$ increases. However, the power-law distribution of weak forces observed in polydisperse systems [28] or for particles of nonspherical shapes at high anisotropy [17] cannot directly be captured by this model. Such power-law distributions suggest a self-similar structure in the range of weak forces so that, in a population-based model as the one introduced here, the population of involved forces should explicitly depend on the force level.

\section{Conclusion}

In this paper, after a brief review of major characteristics of force distribution in granular materials, we first discussed and illustrated the effects of steric exclusions with respect to force balance conditions. We argued that vectorial balance is not efficient for splitting or recombining forces since orientational randomness of contacts at the particle level is suppressed or highly restricted by angular hindrance. For this reason, the proper disorder of a granular assembly lies in the fluctuations of particle environments as a result of connectivity disorder. Hence, force chains occur naturally alongside chains of particles and branch when a contact is missing or they fade out by generating weak forces due to geometrical disorder. In this way, a force $f$ can be generated only from a force $f^{\prime}>f$ in its environment and in the presence of forces $f^{\prime \prime}<f$ required to restore force balance, as inferred from force transfer equations (3).

Inspired by this picture, we formulated a "cascade" model of contact forces that predicts an analytical expression (10) of force density function with a single free parameter. This expression provides a remarkably good fit of both numerical and experimental data of force densities and correctly predicts the exponential falloff of strong forces, the small peak observed below the mean force and the non-zero probability of vanishingly small forces. Such a robust agreement with an unmonotonic behavior provides strong credit for the underlying physical mechanism of a cascade-like process in which the 
probability of a contact force is conditioned by those of larger and lower forces. The statistics of force populations in contact environments obtained by numerical simulations supports this description and will be published elsewhere.

\section{References}

[1] P. Dantu, Contribution à l'étude mécanique et géométrique des milieux pulvérulents, in: Proc. of the 4th International Conf. on Soil Mech. and Foundation Eng., vol. 1, Butterworths Scientific Publications, London, 1957, pp. 144-148.

[2] A. Drescher, G. de Josselin de Jong, Photoelastic verification of a mechanical model for the flow of a granular material, J. Mech. Phys. Solids 20 (1972) $337-351$.

[3] T. Travers, M. Ammi, D. Bideau, A. Gervois, J.-C. Messager, J.-P. Troadec, Uniaxial compression of 2d packings of cylinders. Effects of weak disorder, Europhys. Lett. 4 (3) (1987) 329-332.

[4] C. Liu, S.R. Nagel, D.A. Schecter, S.N. Coppersmith, S. Majumdar, O. Narayan, T.A. Witten, Force fluctuations in bead packs, Science 269 (1995) 513.

[5] F. Radjai, M. Jean, J.-J. Moreau, S. Roux, Force distributions in dense two-dimensional granular systems, Phys. Rev. Lett. 77 (2) (1996) 274.

[6] C. Thornton, Force transmission in granular media, KONA Powder Part. 15 (1997) 81-90.

[7] O. Tsoungui, D. Vallet, J.-C. Charmet, Use of contact area trace to study the force distributions inside 2d granular systems, Granul. Matter 1 (2) (1998) 65-69.

[8] D.M. Mueth, H.M. Jaeger, S.R. Nagel, Force distribution in a granular medium, Phys. Rev. E 57 (1998) 3164.

[9] G. Lovol, K. Maloy, E. Flekkoy, Force measurements on static granular materials, Phys. Rev. E 60 (1999) $5872-5878$.

[10] F. Radjai, S. Roux, J.J. Moreau, Contact forces in a granular packing, Chaos 9 (3) (1999) 544-550.

[11] S.G. Bardenhagen, J.U. Brackbill, D. Sulsky, Numerical study of stress distribution in sheared granular material in two dimensions, Phys. Rev. E 62 (2000) 3882-3890.

[12] L.E. Silbert, G.S. Grest, J.W. Landry, Statistics of the contact network in frictional and frictionless granular packings, Phys. Rev. E 66 (2002) 1-9.

[13] T.S. Majmudar, R.P. Behringer, Contact force measurements and stress-induced anisotropy in granular materials, Nature 435 (2005) $1079-1082$.

[14] D.L. Blair, N.W. Mueggenburg, A.H. Marshall, H.M. Jaeger, S.R. Nagel, Force distributions in three-dimensional granular assemblies: effects of packing order and interparticle friction, Phys. Rev. E 63 (2001) 041304.

[15] E. Azéma, F. Radjai, R. Peyroux, G. Saussine, Force transmission in a packing of pentagonal particles, Phys. Rev. E 76 (1 Pt 1 ) (2007) 011301.

[16] V. Richefeu, M.S. El Youssoufi, E. Azéma, F. Radjai, Force transmission in dry and wet granular media, Powder Technol. 190 (2009) 258-263.

[17] E. Azéma, G. Saussine, F. Radjai, Quasistatic rheology, force transmission and fabric properties of a packing of irregular polyhedral particles, Mech. Mater. 41 (2009) 729-741.

[18] T. Travers, M. Ammi, D. Bideau, A. Gervois, J.-C. Messager, J.-P. Troadec, Mechanical size effects in 2d granular media, J. Phys. France 49 (1988) 939-948.

[19] O. Tsoungui, D. Vallet, J.-C. Charmet, S. Roux, "Partial pressures" supported by granulometric classes in polydisperse granular media, Phys. Rev. E 57 (4) (1998) 4458-4465.

[20] F. Radjai, D.E. Wolf, The origin of static pressure in dense granular media, Granul. Matter 1 (1998) 3-8.

[21] S.J. Antony, Evolution of force distribution in three-dimensional granular media, Phys. Rev. E 63 (1 Pt 1) (2001) 011302.

[22] F. Radjai, D.E. Wolf, M. Jean, J. Moreau, Bimodal character of stress transmission in granular packings, Phys. Rev. Lett. 80 (1998) 61-64.

[23] C.S. O’Hern, S.A. Langer, A.J. Liu, S.R. Nagel, Force distributions near jamming and glass transitions, Phys. Rev. Lett. 86 (1) (2001) $111-114$.

[24] J.H. Snoeijer, T.J.H. Vlugt, M. van Hecke, W. van Saarloos, Force network ensemble: a new approach to static granular matter, Phys. Rev. Lett. 92 (5) (2004) 054302.

[25] P.T. Metzger, Comment on "Mechanical analog of temperature for the description of force distribution in static granular packings", Phys. Rev. E, Stat. Nonlinear Soft Matter Phys. 69 (5 Pt 1) (2004) 053301, discussion 053302.

[26] P.T. Metzger, Granular contact force density of states and entropy in a modified Edwards ensemble, Phys. Rev. E, Stat. Nonlinear Soft Matter Phys. 70 (5 Pt 1) (2004) 051303.

[27] E. Azéma, F. Radjai, Internal structure of inertial granular flows, Phys. Rev. Lett. 112 (7) (2014) 078001.

[28] C. Voivret, F. Radjai, J.-Y. Delenne, M.E. Youssoufi, Force transmission in polydisperse granular media, Phys. Rev. Lett. 102 (2009) 178001.

[29] B. Saint-Cyr, J.-Y. Delenne, C. Voivret, F. Radjai, P. Sornay, Rheology of granular materials composed of nonconvex particles, Phys. Rev. E 84 (4) (2011) 041302 .

[30] E. Azema, F. Radjai, B. Saint-Cyr, J.-Y. Delenne, P. Sornay, Rheology of 3d packings of aggregates: microstructure and effects of nonconvexity, Phys. Rev. E 87 (2013) 052205.

[31] D.-H. Nguyen, E. Azéma, F. Radjai, P. Sornay, Effect of size polydispersity versus particle shape in dense granular media, Phys. Rev. E 90 (1) (2014) 012202.

[32] E.I. Corwin, H.M. Jaeger, S.R. Nagel, Structural signature of jamming in granular media, Nature 435 (7045) (2005) $1075-1078$.

[33] S.N. Coppersmith, C. Liu, S. Majumdar, O. Narayan, T.A. Witten, Model for force fluctuations in bead packs, Phys. Rev. E 53 (5) (1996) $4673-4685$.

[34] J. Goddard, On entropy estimates of contact forces in static granular assemblies, Int. J. Solids Struct. 41 (21) (2004) $5851-5861$.

[35] R. Youngquist, P. Metzger, K. Kilts, Force density function relationships in 2-d granular media, SIAM J. Appl. Math. 65 (6) (2005) $1855-1869$.

[36] I. Agnolin, J.-N. Roux, Internal states of model isotropic granular packings. I. Assembling process, geometry, and contact networks, Phys. Rev. E, Stat. Nonlinear Soft Matter Phys. 76 (6-1) (2007) 061302.

[37] J. Moreau, Some numerical methods in multibody dynamics: application to granular, Eur. J. Mech. A, Solids 13 (1994) $93-114$.

[38] F. Radjai, V. Richefeu, Contact dynamics as a nonsmooth discrete element method, Mech. Mater. 41 (2009) $715-728$.

[39] F. Radjai, L. Brendel, S. Roux, Nonsmoothness, indeterminacy, and friction in two-dimensional arrays of rigid particle, Phys. Rev. E 54 (1) (1996) 861.

[40] J.H. Snoeijer, T.J.H. Vlugt, W.G. Ellenbroek, M. van Hecke, J.M.J. van Leeuwen, Ensemble theory for force networks in hyperstatic granular matter, Phys. Rev. E, Stat. Nonlinear Soft Matter Phys. 70 (6 Pt 1) (2004) 061306.

[41] F. Radjai, J.-Y. Delenne, É. Azema, S. Roux, Fabric evolution and accessible geometrical states in granular materials, Granul. Matter 14 (2) (2012) $259-264$. 